\title{
Targeting Toll-like receptor 4 prevents cobalt-mediated inflammation
}

\author{
Helen Lawrence ${ }^{1, *}$, Amy Elizabeth Mawdesley, ${ }^{1, *}$, James Patrick Holland², John \\ Andrew Kirby ${ }^{1}$, David John Deehan ${ }^{1,2}$ and Alison Jane Tyson-Capper ${ }^{1}$ \\ ${ }^{1}$ Institute of Cellular Medicine, Newcastle University, Newcastle upon Tyne, UK \\ 2 Musculoskeletal Services, Freeman Hospital, Newcastle upon Tyne, UK \\ * Joint first authors
}

Correspondence to: Alison Tyson-Capper, email: alison.tyson-capper@ncl.ac.uk

Keywords: cobalt, metal-on-metal, antibody, TLR4, inflammation, Immunology and Microbiology Section, Immune response, Immunity

Received: December 16,2015 Accepted: January 23, $2016 \quad$ Published: January 31, 2016

\section{ABSTRACT}

Cobalt-chrome alloy is a widely used biomaterial in joint replacements, dental implants and spinal rods. Although it is an effective and biocompatible material, adverse reactions to metal debris (ARMD) have arisen in a minority of patients, particularly in those with metal-on-metal bearing hip replacements. There is currently no treatment for ARMD and once progressive, early revision surgery of the implant is necessary. Therapeutic agents to prevent, halt or reverse ARMD would therefore be advantageous.

Cobalt ions activate Toll-like receptor 4 (TLR4), an innate immune receptor responsible for inflammatory responses to bacterial lipopolysaccharide (LPS) resulting in the production of pro-inflammatory cytokines and chemokines. We hypothesised that anti-TLR4 neutralising antibodies, reported to inhibit TLR4mediated inflammation, could prevent the inflammatory response to cobalt ions in an in vitro macrophage cell culture model.

This study shows that a monoclonal anti-TLR4 antibody inhibited cobalt-mediated increases in pro-inflammatory IL8, CCL20 and IL1A expression, as well as IL-8 secretion. In contrast, a polyclonal antibody did not prevent the effect of cobalt ions on either IL-8 or IL1A expression, although it did have a small effect on the CCL20 response. Interestingly, both antibodies inhibited cobalt-mediated neutrophil migration although the greater effect was observed with the monoclonal antibody.

In summary our data shows that a monoclonal anti-TLR4 antibody can inhibit cobalt-mediated inflammatory responses while a polyclonal antibody only inhibits the effect of specific cytokines. Anti-TLR4 antibodies have therapeutic potential in ARMD although careful antibody design is required to ensure that the LPS response is preserved.

\section{INTRODUCTION}

Cobalt-chrome alloy is widely used as a biomaterial in orthopaedic joint replacements, spinal reconstructive rods and dental implants. Over recent years concerns have emerged regarding early failure in hip replacements with cobalt-chrome metal-on-metal (MoM) bearings. These failing joint replacements are associated with high levels of cobalt and chromium in the blood and within the joint space itself. Clinical reactions include abnormal fluid collections, metallosis, muscle and soft tissue necrosis, pseudotumours, osteolysis and implant loosening [1]. These tissue responses are collectively termed 'adverse reactions to metal debris' (ARMD) [2]. Analysis of the MoM peri-implant tissues reveals a cellular infiltrate dominated by macrophages [3] and lymphocytes [4] along with high levels of cytokines and chemokines which strongly suggests an inflammatory response.

Currently MoM total hip replacement revision rates range from $15 \%$ to $35 \%$ at 7 years compared to $2 \%$ for 
conventional metal-on-polyethylene devices [5]. Despite the high rate of revision for MoM hip replacements there are still more than 1 million patients worldwide with these hips implanted and un-revised [6], which require ongoing surveillance. The major therapeutic option for pseudotumours and ARMD is currently removal of all cobalt-chrome alloy and revision to a ceramic or polyethylene hip implant. This is a more complex surgical procedure than primary hip replacements due to the erosion bone stock and loss of muscle and soft tissue, and post-revision recovery is often slow and incomplete. Hip revision for pseudotumours has been associated with operative risks comprising 75\% blood transfusion, 19\% recurrent dislocation, 19\% nerve palsy, 13\% component loosening and $40 \%$ risk of re-revision [7, 8] causing distress to patients and a considerable financial burden to the NHS. An adjuvant therapeutic agent that could ameliorate or inhibit the initiation of the inflammatory signalling cascade that results in ARMD and thus lessen the surgical insult or even dampen down ARMD without the need for surgical intervention would therefore be attractive. Current research into ARMD therapies focuses on modulation of the ongoing inflammatory response by polarising macrophages towards an antiinflammatory phenotype [9] or inhibiting the function of pro-inflammatory cytokines such as CCL2 [10] and have shown positive results in in vivo models. However they are made more complex by the pleiotropic and redundant nature of cytokines and chemokines.

Recent studies by our group and others have shown that cobalt ions generated by MoM hip articulation can activate the innate immune receptor Toll-like receptor 4 (TLR4) [11-14] which is most commonly known as the receptor for Gram negative bacterial lipopolysaccharide (LPS). Cobalt-mediated TLR4 activation increases the secretion of pro-inflammatory cytokines such as interleukin-8 (IL-8, chemokine (C-X-C motif) ligand 8 or CXCL8), interleukin-6 (IL-6) and chemokine (C-X-C motif) ligand 10 (CXCL10) in macrophages and endothelial cells $[11,14]$. A small molecule TLR4 antagonist, CLI-095, is able to prevent these responses [14] but its therapeutic use is limited because of its intracellular mechanism of action.

Antibodies represent a successful and rapidly evolving therapeutic strategy. Both monoclonal and polyclonal anti-TLR4 antibodies are currently under investigation as therapeutic agents in the prevention of septic shock induced by LPS [15], and a number of antiTLR4 antibodies are currently in clinical trials to improve test their effectiveness in diseases including rheumatoid arthritis [16]. We therefore hypothesised that anti-TLR4 neutralising antibodies could prevent cobalt-mediated TLR4 activation, using changes in cytokine secretion and expression by macrophages as markers of receptor activation and inhibition.

\section{RESULTS}

\section{Antibody cytotoxicity}

MonoMac 6 cells were incubated with $10 \mu \mathrm{g} /$ $\mathrm{ml} \mathrm{MAb2-hTLR4} \mathrm{or} 5 \mu \mathrm{g} / \mathrm{ml}$ PAb-hTLR4 for $16 \mathrm{~h}$. Cytotoxicity was assessed by trypan blue staining and cells were counted using a Luna II automated cell counter. MAb2-hTLR4 did not affect MonoMac 6 cells viability (Figure 1) while PAb-hTLR4 decreased cell viability from $100 \%$ to $94 \%$ (Figure 1 ).

\section{Inhibition of cobalt-mediated inflammatory responses by a monoclonal anti-TLR4 antibody}

TLR4-expressing MonoMac 6 cells have previously been shown to upregulate IL-8 secretion and expression when challenged with cobalt ions [14] and were therefore selected as an in vitro cell model for this study, using IL-8 as a marker of TLR4 activation. MonoMac 6 cells were pre-treated with $10 \mu \mathrm{g} / \mathrm{ml}$ MAb2-hTLR4 for $1 \mathrm{~h}$ prior to $16 \mathrm{~h}$ stimulation with $0.75 \mathrm{mM} \mathrm{CoCl}_{2}$ or $100 \mathrm{ng} /$ ml LPS. IL-8 protein levels were measured by ELISA and $I L 8$ expression was quantified by qRT-PCR. $\mathrm{CoCl}_{2}$ and LPS both significantly increased IL- 8 secretion to approximately 5000pg/ml $(\mathrm{p}<0.001)$ (Figure 2A). Pretreatment with MAb2-hTLR4 significantly decreased IL-8 secretion to $3000 \mathrm{pg} / \mathrm{ml}$ in $\mathrm{CoCl} 2$-stimulated cells $(p<0.001)$ and $4000 \mathrm{pg} / \mathrm{ml}$ in LPS-stimulated cells. IL 8 gene expression followed a similar pattern with significant upregulation in expression following $\mathrm{CoCl}_{2}$ and LPS stimulation (both $p<0.001$ ) (Figure 2B). MAb2-hTLR4 significantly inhibited $\mathrm{CoCl}_{2}$-mediated IL8 expression, reducing it from 20 -fold to 5 -fold $(p<0001)$. The monoclonal antibody also prevented the IL 8 expression increase induced by LPS $(p<0.001)$.

\section{A polyclonal anti-TLR4 antibody does not prevent cobalt-mediated IL-8 changes}

A polyclonal anti-TLR4 neutralising antibody, PAbhTLR4, is reported to inhibit inflammatory responses to TLR4 ligands. Using IL-8 expression and secretion by MonoMac 6 cells as a marker of inflammation, the ability of PAb-hTLR4 to prevent the cellular response to cobalt was assessed. MonoMac 6 cells were pre-treated with $5 \mu \mathrm{g} / \mathrm{ml} \mathrm{PAb}-\mathrm{hTLR} 4$ for 10 minutes prior to $16 \mathrm{~h}$ stimulation with either $0.75 \mathrm{mM} \mathrm{CoCl}$ or $100 \mathrm{ng} / \mathrm{ml} \mathrm{LPS}$. IL- 8 secretion was measured by ELISA and expression by qRT-PCR.

PAb-hTLR4 did not inhibit $\mathrm{CoCl}_{2}$ or LPS-mediated IL-8 secretion (Figure 2C) ( $p>0.4$ in both cases). In untreated cells there was an increase in IL-8 secretion in 

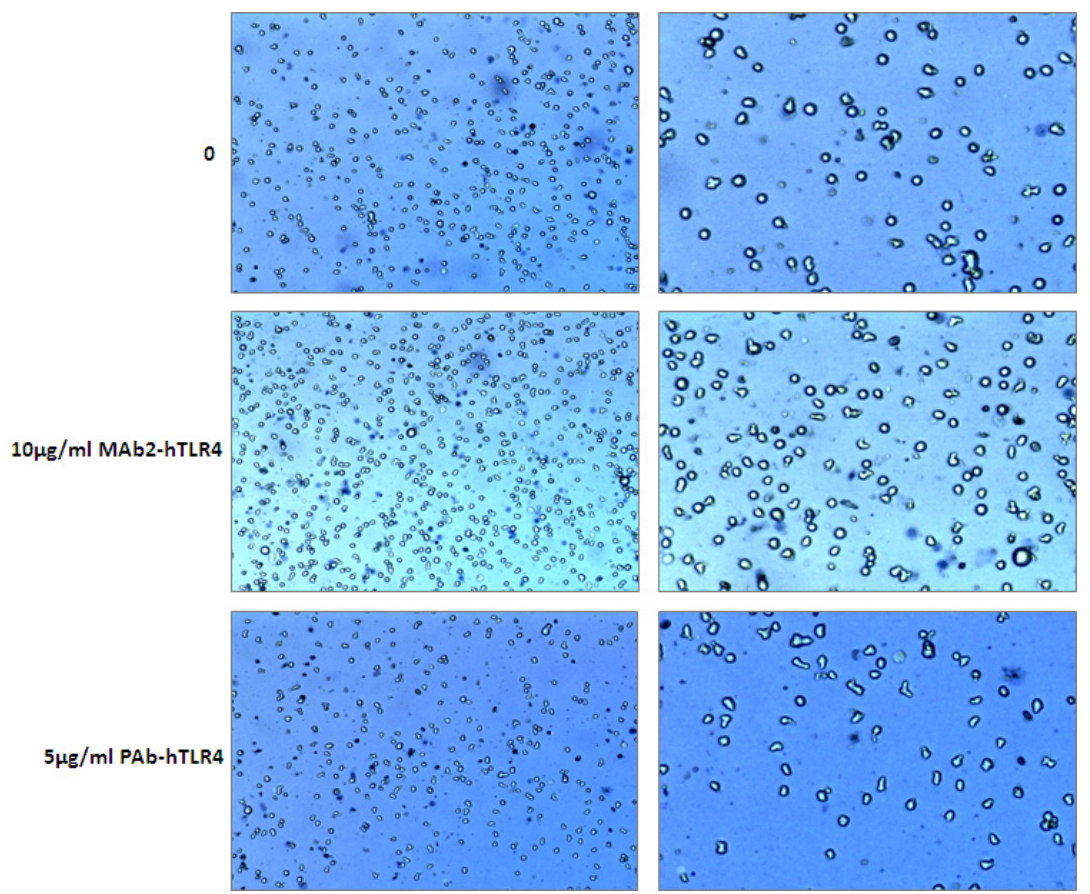

Figure 1: Cytotoxicity assay for MAb2-hTLR4 and PAb-hTLR4. MonoMac 6 cells were incubated with 10 $\mu \mathrm{g} / \mathrm{ml}$ MAb2-hTLR4 or $5 \mu \mathrm{g} / \mathrm{ml} \mathrm{PAb-hTLR} 4$ for $16 \mathrm{~h}$. A cytotoxicity assay was conducted using trypan blue staining and cell counting on a Luna II automated cell counter. Cell viability was 100\% in untreated and MAb2-hTLR4-treated cells, and 94\% in those incubated with PAb-hTLR4. Images on the right are magnified versions of those on the left.

A.

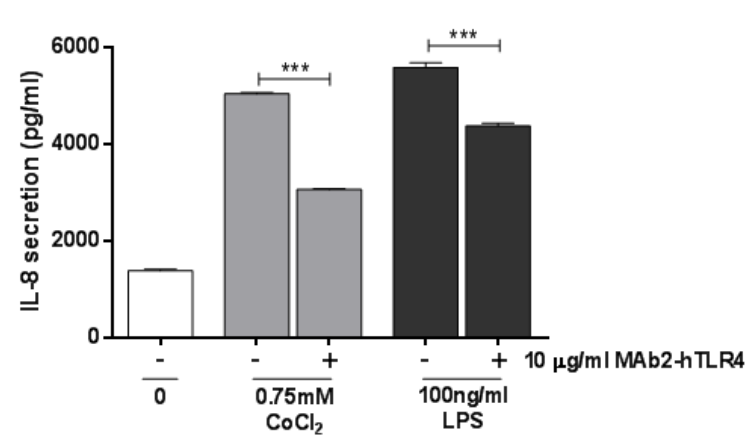

c.

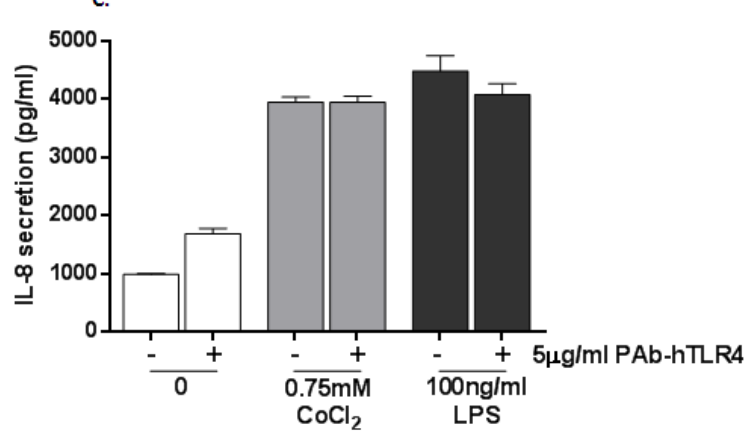

B.
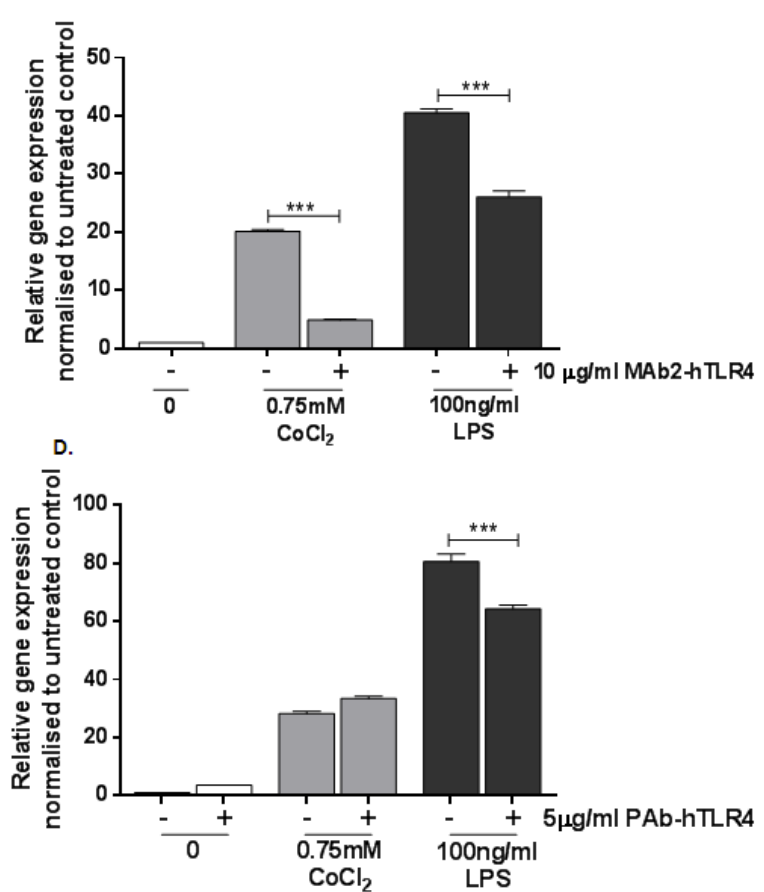

Figure 2: Effect of monoclonal and polyclonal anti-TLR4 neutralising antibody on cobalt-mediated IL-8 expression and secretion. A. MonoMac 6 cells were pre-incubated with $10 \mu \mathrm{g} / \mathrm{ml}$ MAb2-hTLR4 for $1 \mathrm{~h}$ and then stimulated with $0.75 \mathrm{mM} \mathrm{CoCl}_{2}$ or $100 \mathrm{ng} / \mathrm{ml}$ LPS for $16 \mathrm{~h}$. Supernatant was collected and IL-8 secretion was measured by ELISA. B. qRT-PCR was performed to evaluate the effect of MAb2-hTLR4 on $I L 8$ expression. C. MonoMac 6 cells were pre-incubated with $5 \mu \mathrm{g} / \mathrm{ml} \mathrm{PAb-hTLR4} \mathrm{for} 10$ minutes followed by stimulation with $0.75 \mathrm{mM} \mathrm{CoCl}_{2}$ or $100 \mathrm{ng} / \mathrm{ml}$ LPS for $16 \mathrm{~h}$. Supernatant was collected and IL-8 secretion measured by ELISA. D. qRTPCR was performed to evaluate the effect of PAb-hTLR4 on IL 8 expression. Data is representative of three independent experiments and statistical significance was calculated by one-way analysis of variance (ANOVA) with Tukey's test for multiple comparisons comparing all samples to each other. 
the presence of the antibody although this did not reach statistical significance $(p=0.051)$. IL 8 expression following LPS stimulation was inhibited by PAb-hTLR4 $(p<0.001)$ (Figure 2D). However the antibody could not prevent the effect of cobalt ions on IL8 expression $(p=0.095)$.

\section{MAb2-hTLR4 prevents cobalt-mediated expression of $C C L 20$ and $I L 1 A$}

Using qRT-PCR arrays 4h stimulation of MonoMac 6 cells with $0.75 \mathrm{mM} \mathrm{CoCl}{ }_{2}$ was found to upregulate expression of pro-inflammatory CCL20 and ILIA. The array results were subsequently validated by TaqManbased qRT-PCR. This analysis confirmed a significant upregulation in both CCL20 (Figure 3A) and IL1A (Figure 3B) expression following $4 \mathrm{~h}$ exposure of MonoMac 6 cells to $0.75 \mathrm{mM} \mathrm{CoCl}_{2}$ (both $p<0.001$ ), showing the potential of these genes as targets for studying antibody efficacy.

The ability of MAb2-hTLR4 to inhibit cobaltmediated changes in CCL2O and IL1A expression was therefore investigated using the same treatment protocol as described for the IL-8 assays. CCL20 expression was upregulated approximately 10 -fold by $\mathrm{CoCl}_{2}$ stimulation $(p<0.001)$ whilst the effect of LPS was greater, with a 50 fold increase in expression $(p<0.001)$ (Figure 4A). MAb2hTLR4 significantly decreased expression of CCL20 in response to $\mathrm{CoCl} 2$ and the positive control LPS $(p<0.001$ in both cases). ILIA expression was also significantly increased by $\mathrm{CoCl}_{2}(p=0.0283)$ and LPS $(p<0.001)$ (Figure $4 \mathrm{~B})$. In the presence of MAb2-hTLR4 $\mathrm{CoCl}_{2}$-mediated IL1A expression was significantly decreased $(p=0.0224)$ as was that of LPS $(p<0.001)$.

\section{A polyclonal anti-TLR4 antibody can inhibit other cobalt-mediated inflammatory effects}

Expression of CCL20 and IL1A was investigated to validate the inability of PAb-hTLR4 to inhibit TLR4 activation by cobalt ions using the same treatment protocol described for the IL-8 assays. CCL20 expression was assessed by qRT-PCR and found to be abrogated by the polyclonal antibody (Figure 4C) $(p<0.001)$ but there was no effect on ILIA (Figure 4D) ( $p=0.8246)$. PAb-hTLR4 inhibited expression of both genes in response to LPS stimulation $(p<0.001$ for $C C L 20$ and $\mathrm{p}=0.0044$ for $I L 1 A)$.

\section{Cobalt-mediated neutrophil migration is inhibited by TLR4 neutralising antibodies}

Inflammatory cell infiltration is a hallmark of MoM peri-implant tissues. As IL-8 secretion is significantly increased by cobalt ions and IL- 8 is chemotactic for neutrophils, the effect of cobalt and the neutralising antibodies on primary neutrophil migration was assessed.
MonoMac 6 cells were pre-stimulated with MAb2-hTLR4 or PAb-hTLR4 as described earlier before 16h stimulation with $0.75 \mathrm{mM} \mathrm{CoCl}_{2}$ or $100 \mathrm{ng} / \mathrm{ml}$ LPS. Conditioned media was collected and used in a transwell neutrophil migration assay.

Conditioned media from cobalt-stimulated MonoMac 6 cells significantly increased neutrophil migration $(p<0.001)$ (Figure 5). Both MAb2-hTLR4 and PAb-hTLR4 pre-incubation significantly inhibited this effect (both $p<0.001$ ) although the monoclonal antibody induced a greater reduction in migration than the polyclonal.

\section{DISCUSSION}

This study demonstrates that a monoclonal antiTLR4 neutralising antibody (MAb2-hTLR4) inhibits pro-inflammatory IL8,ILIA and CCL20 expression and IL-8 secretion by MonoMac 6 cells following cobalt stimulation. MAb2-hTLR4 provided more effective inhibition than the polyclonal antibody PAb-hTLR4 which did not prevent cobalt-mediated IL- 8 or $I L 1 A$ responses although it did inhibit CCL20 expression. Despite the differences in their inhibitory effect both neutralising antibodies prevented neutrophil migration in response to cobalt stimulation, with a greater effect observed for the monoclonal antibody.

The differences in inhibition between the monoclonal and polyclonal antibodies are likely due to the fact that cobalt and LPS bind to TLR4 in different ways; cobalt binds directly to the receptor within a 'histidine pocket' composed of histidine residues H431, 456 and 458 [11] while LPS initially binds LPS binding protein (LBP) before transfer across a series of adaptor proteins to the receptor itself. If an antibody blocks an adaptor protein binding site then it may inhibit LPS without having an effect on the cobalt response. However as PAb-hTLR4 blocked CCL20 expression it may have a small effect on receptor homodimerisation which is required for activation by both LPS and cobalt ions [11].

The differences in cobalt and LPS binding sites could be exploited for the development of an antibody to prevent ARMD. For an anti-TLR4 antibody to be successful in preventing the inflammatory response to cobalt ions it is essential that the immunological response to LPS is maintained or else there is a high risk of overwhelming bacterial infection and potentially lethal septic shock. The development of a novel antibody targeting the cobalt-specific binding site of TLR4 could prevent activation by cobalt ions while still allowing an inflammatory response to LPS.

This study focused on a select group of proinflammatory cytokines and chemokines induced by cobalt based on previous work performed by the group. However individual cytokine targets are of limited value when investigating the potential of a therapeutic agent as it is the 
A.

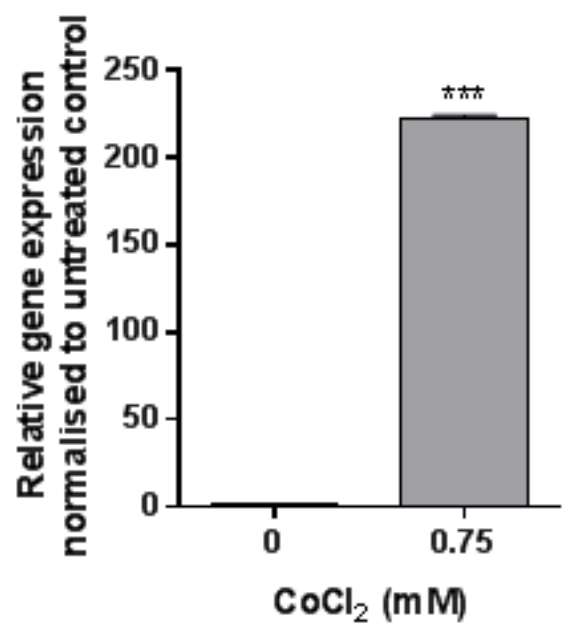

B.

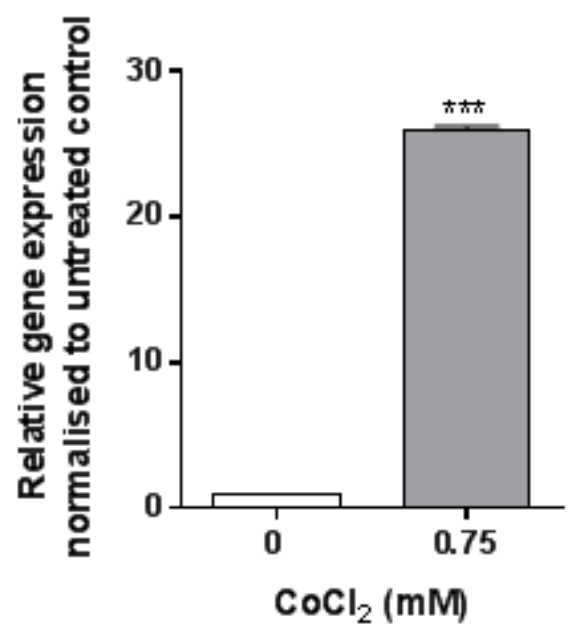

Figure 3: CCL20 and IL1A expression following $\mathrm{CoCl}_{2}$ stimulation. MonoMac 6 cells were stimulated with $0.75 \mathrm{mM} \mathrm{CoCl}_{2}$ for $4 \mathrm{~h}$ before qRT-PCR was conducted to assess A. CCL20 and B. IL1A expression. Data is representative of three independent experiments and statistical significance was calculated by Student's $t$ test.
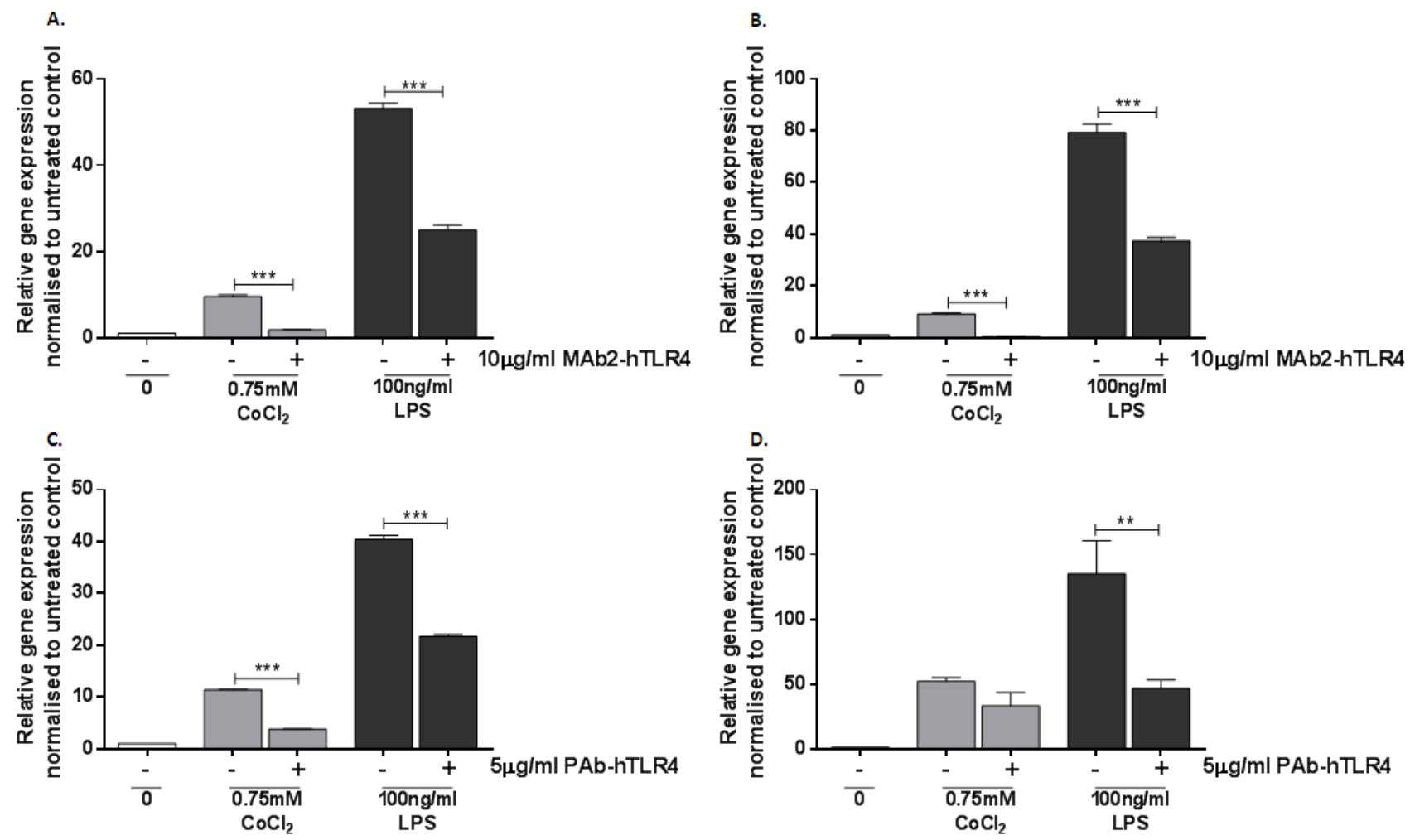

Figure 4: Effect of MAb2-hTLR4 and PAb-hTLR4 on cobalt-mediated CCL20 and IL1A expression. A. \& B. MonoMac 6 cells were pre-treated with $10 \mu \mathrm{g} / \mathrm{ml}$ MAb2-hTLR4 for 1 hour prior to $16 \mathrm{~h}$ stimulation with $0.75 \mathrm{mM} \mathrm{CoCl}{ }_{2}$ or $100 \mathrm{ng} / \mathrm{ml}$ LPS. RNA was isolated and cDNA synthesised by reverse transcription. CCL20 (A) and IL1A (B) expression was quantified by qRT-PCR. C\&D. MonoMac 6 cells were pre-treated with $5 \mu \mathrm{g} / \mathrm{ml}$ PAb-hTLR4 for 10 minutes before $16 \mathrm{~h}$ stimulation with $0.75 \mathrm{mM} \mathrm{CoCl}$ or $100 \mathrm{ng} / \mathrm{ml}$ LPS. RNA was isolated and cDNA synthesised by reverse transcription. CCL20 C. and IL1A D. expression was quantified by qRT-PCR. Data is representative of three independent experiments and statistical significance was calculated by one-way ANOVA with Tukey's test for multiple comparisons comparing all samples to each other. 


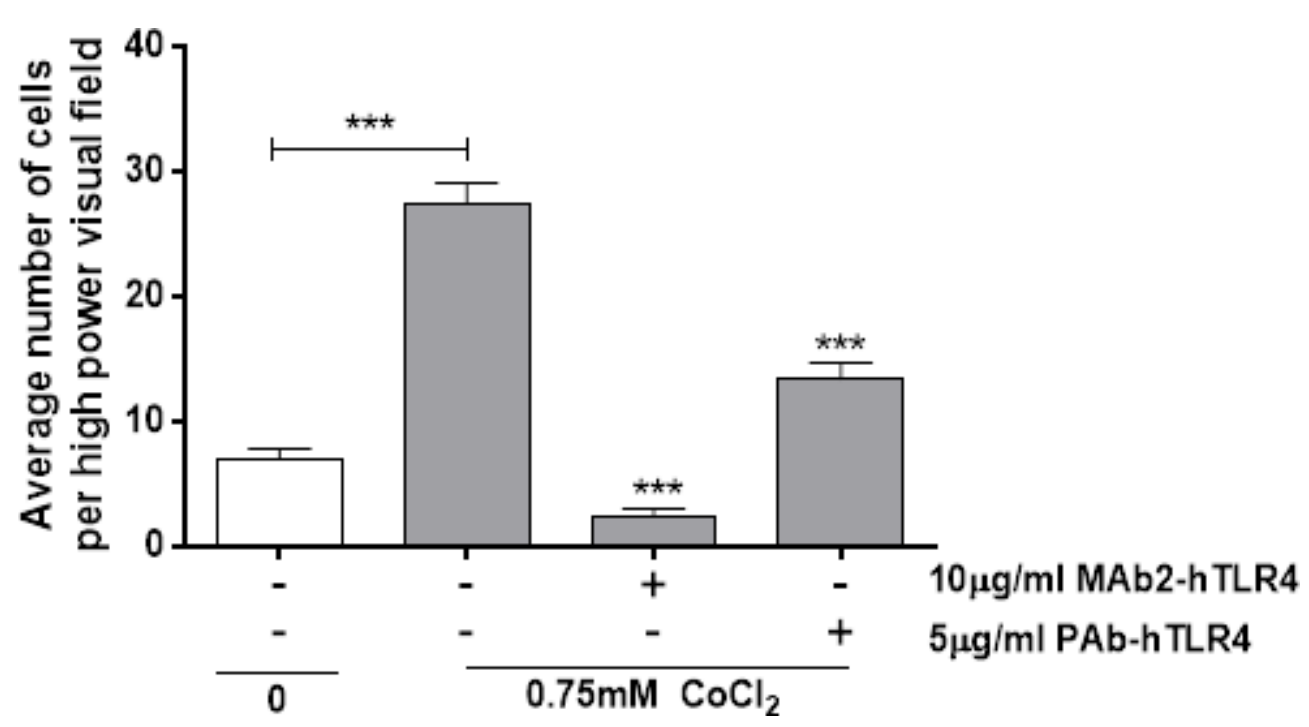

Figure 5: Anti-TLR4 neutralising antibodies inhibit cobalt-mediated neutrophil chemotaxis. MonoMac 6 cells were pretreated with $10 \mu \mathrm{g} / \mathrm{ml} \mathrm{MAb2-hTLR} 4$ for 1 hour or $5 \mu \mathrm{g} / \mathrm{ml}$ PAb-hTLR4 for 10 minutes before $16 \mathrm{~h}$ stimulation with $0.75 \mathrm{mM} \mathrm{CoCl}_{2}$ or $100 \mathrm{ng} /$ $\mathrm{ml}$ LPS. Supernatant was collected and chemotactic properties assessed using a transwell neutrophil migration assay. Data is representative of three independent experiments and statistical significance was calculated by one-way ANOVA with Tukey's test for multiple comparisons comparing all samples to each other.

cumulative effect of cytokines and chemokines that leads to ARMD. For this reason neutrophil migration assays were performed to assess the capacity of the antibodies to prevent cobalt-mediated neutrophil recruitment. Both antibodies inhibited neutrophil migration; the effect of the monoclonal antibody was greater than that of the polyclonal which supports the other data described in this study. It also indicates that neutrophil chemokines other than IL-8 are inhibited by PAb-hTLR4. Additional in vitro functional assays or in an in vivo mouse model with humanised TLR4 could be used to further investigate these effects and antibody efficacy.

In summary, our data shows that anti-TLR4 neutralising antibodies can prevent cobalt activation of the receptor and subsequent inflammatory cytokine upregulation by human macrophages. The design of an antibody for ARMD therapy will be critical to provide the most effective inhibition of cobalt-mediated inflammation while still ensuring that the LPS-binding capacity of TLR4 is preserved. This work suggests that TLR4 has excellent potential as a therapeutic target in the prevention of ARMD.

\section{MATERIALS AND METHODS}

\section{MonoMac 6 cells}

MonoMac 6 cells are a TLR4-expressing human macrophage cell line derived from acute monocytic leukaemia. MonoMac 6 cells were cultured in complete RPMI-1640 medium supplemented with $10 \%$ foetal bovine serum (FBS), 2mM L-glutamine, $50 \mathrm{~d} / \mathrm{ml}$ penicillin and $50 \mu \mathrm{g} / \mathrm{ml}$ streptomycin (all Sigma-Aldrich, Gillingham, UK).

\section{Metal ion stimulation}

Cobalt chloride hexahydrate $\left(\mathrm{CoCl}_{2}\right)$ was diluted in complete cell culture medium and used at $0.75 \mathrm{mM}$, a physiological concentration optimised in a previous study [14]. TLR4-specific LPS (from E. coli serotype J5, Alexis Biochemicals, San Diego, USA) was used at $100 \mathrm{ng} / \mathrm{ml}$ as a positive control for receptor activation.

\section{Neutralising antibodies}

PAb-hTLR4 (Invivogen, San Diego, USA) is a polyclonal rat IgG antibody reported to neutralise TLR4 and prevent activation by its ligands [17]. MAb2-hTLR4 (Invivogen) is a monoclonal mouse IgG1 antibody (clone $3 \mathrm{C} 3$ ) reported to neutralise TLR4 and prevent receptor activation by agonists [15].

\section{Cytotoxicity}

Cytotoxicity was assessed by trypan blue staining; $10 \mu 1$ trypan blue dye (Logos Biosystems, Anyang-City, South Korea) was mixed with $10 \mu \mathrm{l}$ cell suspension and the number of live and dead cells was counted using a Luna-II Automated Cell Counter (Logos Biosystems). Data was normalised to $100 \%$ viability for untreated cells. 


\section{Quantitative real-time PCR (qRT-PCR)}

RNA was isolated using a Qiagen RNeasy Mini kit (Qiagen, Venlo, Netherlands) and cDNA synthesized using Superscript III reverse transcriptase (ThermoFisher Scientific, Massachusetts, USA). qRT-PCR was performed using TaqMan gene expression probes (ThermoFisher Scientific). Each reaction contained $5 \mu 1$ TaqMan Gene Expression Mastermix (ThermoFisher Scientific), $8 \mu 1$ diluted cDNA, $2.5 \mu 1$ nuclease-free $\mathrm{H}_{2} \mathrm{O}$ and $0.5 \mu \mathrm{l}$ TaqMan Gene Expression Assay. No-template controls containing $8 \mu 1 \mathrm{H}_{2} \mathrm{O}$ instead of cDNA were included for all assays. All target genes were normalised to GAPDH as a housekeeping gene. Reactions were performed in triplicate.

\section{ELISA}

Enzyme-linked immunosorbent assays (ELISA) were used to quantify cytokine secretion by MonoMac 6 cells. IL-8 secretion was quantified using a Human IL-8 ELISA kit (Peprotech, London, UK) as described previously [14].

\section{Transwell neutrophil migration assay}

Neutrophils were isolated from the whole blood of healthy volunteers by dextran sedimentation (Dextran T500, Pharmacosmos, Holbaek, Denmark) and centrifugation on Percoll (GE Healthcare, Buckinghamshire, UK) density gradients as previously described [18].

A 24-well companion plate (VWR International, Pennsylvania, USA) was blocked with $1 \%$ bovine serum albumin (BSA) (Sigma Aldrich) for 1 hour after which $800 \mu$ l conditioned media from stimulated MonoMac 6 cells was added to each well. A cell culture insert with $3 \mu \mathrm{m}$ pore filter (VWR International) was placed in each well and 500,000 neutrophils added to the upper chamber of each insert. The plate was incubated at $37^{\circ} \mathrm{C}$ for 2 hours to allow for cell migration. Excess media was removed and filters incubated overnight in $100 \%$ methanol. Filters were stained with haematoxylin for 30 minutes and washed in Scott's tap water for 10 minutes. This was followed by dehydration in increasing ethanol concentrations $(50,75$, 90 and $100 \%$ ) for 2 minutes each. Filters were air-dried for a minimum of three hours and were excised and mounted using DPX mountant. Adhered neutrophils were counted at $\times 40$ magnification.

\section{Statistical analysis}

Statistical analysis was performed using GraphPad Prism 6.0 (GraphPad Software Inc., San Diego, USA).
All error bars show the standard error of the mean (SEM) unless otherwise stated. The analysis method is described for each individual experiment. Statistical significance is shown as follows $*=\mathrm{p}<0.05, * *=\mathrm{p}<0.01, * * *=\mathrm{p}<0.001$.

\section{ACKNOWLEDGMENTS}

The authors acknowledge Mr Jonathan Scott for his help with neutrophil isolation.

\section{CONFLICTS OF INTEREST}

The authors have no conflicts of interest to declare.

\section{GRANT SUPPORT}

HL is supported by a studentship from the Newcastle NIHR Biomedical Research Centre and DePuy Synthes Ltd.

\section{REFERENCES}

1. Daniel J, Holland J, Quigley L, Sprague S and Bhandari M. Pseudotumors associated with total hip arthroplasty. Journal of Bone and Joint Surgery - Series A. 2012; 94:86-93.

2. Langton DJ, Jameson SS, Joyce TJ, Hallab NJ, Natu S and Nargol AVF. Early failure of metal-on-metal bearings in hip resurfacing and large-diameter total hip replacement: A consequence of excess wear. Journal of Bone and Joint Surgery - Series B. 2010; 92:38-46.

3. Perino G, Ricciardi BF, Jerabek SA, Martignoni G, Wilner G, Maass D, Goldring SR and Purdue PE. Implant based differences in adverse local tissue reaction in failed total hip arthroplasties: A morphological and immunohistochemical study. BMC Clinical Pathology. 2014; 14:39.

4. Watters TS, Cardona DM, Menon KS, Vinson EN, Bolognesi MP and Dodd LG. Aseptic lymphocytedominated vasculitis-associated lesion: A clinicopathologic review of an underrecognized cause of prosthetic failure. American Journal of Clinical Pathology. 2010; 134:886893.

5. Powers-Freeling L. (2015). National Joint Registry 12th Annual Report. In: National Joint Registry for England W, Northern Ireland and the Isle of Man, ed.

6. AAOS. (2012). Current concerns with metal-on-metal hip arthroplasty. American Academy of Orthopaedic Surgeons (AAOS)).

7. Grammatopoulos G, Pandit H, Kwon YM, Gundle R, McLardy-Smith P, Beard DJ, Murray DW and Gill HS. Hip resurfacings revised for inflammatory pseudotumour have a poor outcome. Journal of Bone and Joint Surgery - Series B. 2009; 91:1019-1024.

8. De Steiger RN, Miller LN, Prosser GH, Graves SE, Davidson DC and Stanford TE. Poor outcome of revised 
resurfacing hip arthroplasty: 397 cases from the Australian Joint Replacement Registry. Acta Orthopaedica. 2010; 81:72-76.

9. Pajarinen J, Tamaki Y, Antonios JK, Lin TH, Sato T, Yao Z, Takagi M, Konttinen YT and Goodman SB. Modulation of mouse macrophage polarization in vitro using IL-4 delivery by osmotic pumps. Journal of Biomedical Materials Research - Part A. 2015; 103:1339-1345.

10. Jiang X, Sato T, Yao Z, Keeney M, Pajarinen J, Lin Th, Loi F, Egashira K, Goodman S and Yang F. Local delivery of mutant CCL2 protein-reduced orthopaedic implant wear particle-induced osteolysis and inflammation in vivo. Journal of Orthopaedic Research. 2015.

11. Raghavan B, Martin SF, Esser PR, Goebeler M and Schmidt M. Metal allergens nickel and cobalt facilitate TLR4 homodimerization independently of MD2. EMBO Reports. 2012; 13:1109-1115.

12. Tyson-Capper AJ, Lawrence H, Holland JP, Deehan DJ and Kirby JA. Metal-on-metal hips: Cobalt can induce an endotoxin-like response. Annals of the Rheumatic Diseases. 2013; 72(3):460-461.

13. Potnis PA, Dutta DK and Wood SC. Toll-like receptor 4 signaling pathway mediates proinflammatory immune response to cobalt-alloy particles. Cellular Immunology. 2013; 282:53-65.

14. Lawrence H, Deehan D, Holland J, Kirby J and TysonCapper A. The immunobiology of cobalt: Demonstration of a potential aetiology for inflammatory pseudotumours after metal-on-metal replacement of the hip. Bone and Joint Journal. 2014; 69B:1172-1177.

15. Lima CX, Souza DG, Amaral FA, Fagundes CT, Rodrigues IPS, Alves-Filho JC, Kosco-Vilbois M, Ferlin W, Shang L, Elson $\mathrm{G}$ and Teixeira MM. Therapeutic effects of treatment with anti-TLR2 and anti-TLR4 monoclonal antibodies in polymicrobial sepsis. PLoS ONE. 2015; 10.

16. Monnet E, Shang L, Lapeyre G, deGraaf K, Hatterer E, Buatois V, Elson G, Ferlin W, Gabay C, Sokolove J, Jone S, Choy E, McInnes I, Kosco-Vilbois M and de Min C. NI0101, a Monoclonal Antibody Targeting Toll Like Receptor 4 (TLR4) Being Developed for Rheumatoid Arthritis (RA) Treatment with a Potential for Personalized Medicine. Annals of the Rheumatic Diseases. 2015; 74.

17. Ekaney ML, Otto GP, Sossdorf M, Sponholz C, Boehringer M, Loesche W, Rittirsch D, Wilharm A, Kurzai O, Bauer M and Claus RA. Impact of plasma histones in human sepsis and their contribution to cellular injury and inflammation. Critical Care 2014; 18:543.

18. Dransfield I, Buckle AM, Savill JS, McDowall A, Haslett $\mathrm{C}$ and Hogg N. Neutrophil apoptosis is associated with a reduction in CD16 (FcrRIII) expression. Journal of Immunology. 1994; 153:1254-1263. 